\title{
THE LINEWIDTH-LUMINOSITY RELATION FOR BLUE GALAXIES AT A REDSHIFT OF 0.25
}

\author{
HANS-WALTER RIX \\ MPIA, Garching and Steward Observatory \\ MATTHEW COLLESS \\ MSSSO, Australian National University \\ AND \\ PURAGRA GUHATHAKURTA \\ Lick Observatory, UC Santa Cruz
}

\section{Introduction}

This is a progress report on a collaborative effort to probe galaxy evolution by constraining the central mass-to-light ratios of distant field galaxies. Details can be found in a forthcoming paper (Rix, Guhathakurta, Colless \& Ing 1996, RGCI). At the moment, the program is focused on the question of how over-luminous the abundant blue field galaxies at $z \sim 0.3$ are (see Koo, K. and Kron, R. 1992), compared to galaxies of the same circular velocity and type at $z \sim 0$. We have measured the integrated [OII] linewidths for a complete sample of distant blue galaxies to establish a linewidthluminosity-color relation for this population and to compare this relation to the one for local galaxies. In addition, we explore practical strategies to obtain and analyze data so as to best relate the observable quantities (e.g. linewidths) to the physical quantities of interest (such as $v_{\text {circ }}$ or $\mathrm{M} / \mathrm{L}$ ).

\section{Sample and Observations}

The target galaxies were a random subset of LDSS-1 targets (Colless et al. 1993), with magnitudes of $21<m_{b}<22$ and colors $b-r<1.2$. Their integrated emission line spectra for the [OII] doublet were obtained with the AUTOFIB spectrograph at the AAT on the night of October 8, 1993. The spectra covered the redshift range of $0.17<z<0.37$, at a mean spectral resolution of $\sigma_{\text {inst }}=50 \mathrm{~km} / \mathrm{s}$. The $2^{\prime \prime}$ fibres integrate the flux 
over an aperture of $9 \mathrm{kpc} \times\left[\frac{z}{0.25}\right]$ diameter $\left(H_{0}=70 \mathrm{~km} / \mathrm{s} / \mathrm{Mpc}\right)$. Several arguments suggest (see RGCI) that the 24 detected emission lines include all galaxies in the above redshift, magnitude and color range. The emission line widths (LW) were determined by fitting two Gaussians simultaneously to the [OII] doublet, accounting for the instrumental broadening. Most lines were found to be resolved $(\sigma \gtrsim 30 \mathrm{~km} / \mathrm{s})$, but all $\mathrm{LW}$ were $\sigma<100 \mathrm{~km} / \mathrm{s}$. The results of the line fitting for all 24 sample members are displayed in Figure 1.

\section{Data Modeling}

Given their [OII] line luminosity, the LWs observed here are 2-3 times too large to match the relation between the line luminosity and turbulent line width, observed locally for giant Hir regions and "HII galaxies". Hence, the LWs most likely arise from orbital motion and can be related to the circular velocity, $v_{c}$, characterizing the galaxy.

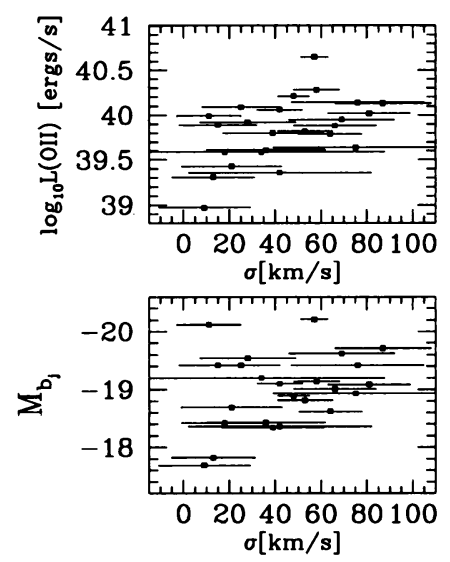

Figure 1. Global galaxy properties versus [OII] linewidth. Integrated [OII] linewidths, $\sigma$, were obtained with $2^{\prime \prime}$ fibres at the AAT for a complete sample of faint $\left(m_{b} \sim 22\right)$, blue $\left(B-V_{\text {rest }}<0.45\right)$ galaxies at $z \sim 0.25$. The three panels compare sigma to the line luminosity, the blue absolute magnitude and the $b-r$ color, respectively. There is a trend for $\sigma$ to increase with $\mathrm{L}([\mathrm{OII}]), M_{b}$ and $b-r$, but the scatter is large. Note that the LW $\sigma$ has not been corrected for inclination.

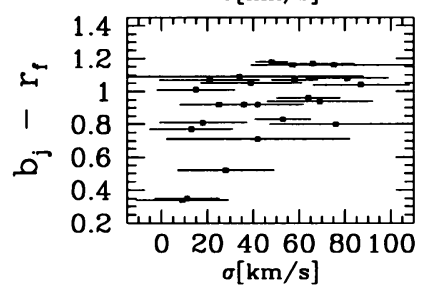

Before we can test with these data whether the linewidth-luminositycolor (LWLC) relation in the distant galaxies differs from the local relation, considerable modeling is required:

(1) How is the observed, integrated (Gaussian) width, $\sigma$, of the emission lines related to the (asymptotic) circular velocity, $v_{c}$, of the galaxy? Aside from their mathematical definition, these two quantities differ because the 
ionized gas has a lumpy asymmetric distribution and because not all of it originates from the "flat" part of the rotation curve. Further, from groundbased data we cannot determine the galaxies' inclinations and must instead assume a distribution in $\cos i$. We try to account for all these effects by using Fabry-Perot data cubes of local galaxies - with similar magnitudes and colors - to simulate the observations of the distant galaxies. The velocity fields of these calibrators were fitted and the emission line flux image was deprojected. Subsequently, the velocity fields and flux images were projected at many random angles and for each the line profile was sampled over an aperture corresponding to the $2^{\prime \prime}$ fibres at $z \sim 0.25$. Gaussians were fit to the line profile and the $\sigma$ was compared to $v_{c}$ obtained from the $2 \mathrm{D}$ velocity field. This procedure leads to the probability distribution, $p\left(\sigma \mid v_{c}\right)$, of observing $\sigma$ with our set-up, given $v_{c}$. This distribution peaks at $\sigma \approx 0.55 v_{c}$ and is quite broad (FWHM $\left.\sim 0.45 v_{c}\right)$. In the subsequent analysis we assume that $p\left(\sigma \mid v_{c}\right)=p\left(\sigma / v_{c}\right)$.
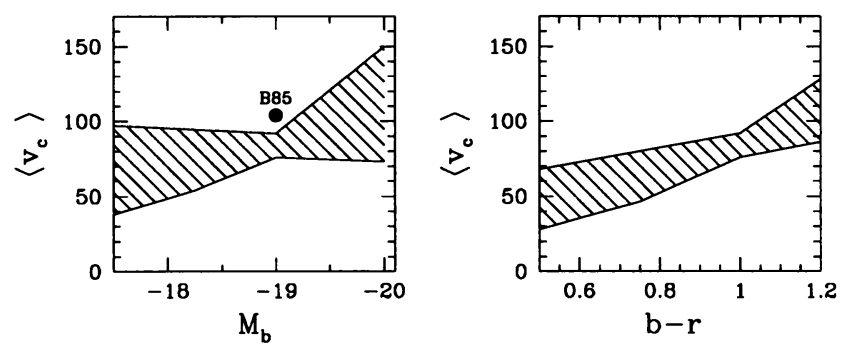

Figure 2. Constraints on the LWLC relation of blue field galaxies at $z \sim 0.25$. The left panel shows the dependence of the mean circular velocity on (blue) luminosity; the two lines enclose the $68 \%$ confidence region from a maximum likelihood analysis. The point B85 indicates the LWL relation for a local sample (Bothun et al. 1985), after reducing $W_{20} / 2 / \sin i$ by $15 \%$ to match $v_{c}$ (optical). The right panel shows the $\left\langle v_{c}\right\rangle$ dependence on color; bluer galaxies have smaller linewidths.

(2) We assume that our distant sample galaxies obey a LWLC relation of the form

$$
\ln \left[v_{c}\left(M_{b}, b-r\right)\right]=\ln \left[v_{c}(-19,1)\right]-\eta\left(M_{b}+19\right)+\zeta[(b-r)-1]
$$

with a fractional velocity scatter of $\Delta$. For each galaxy with $v_{c}\left(M_{b}, b-r\right)$ we should expect to observe $\sigma$ with a probability $p\left(\sigma / v_{c}\right)$. For each combination of parameters $\left[v_{c}(-19,1), \eta, \zeta\right]$ our set of measurements, $\sigma_{o b s}^{i}$ and $\Delta \sigma_{o b s}^{i}$ $(i=1,24)$ defines a likelihood, $\mathcal{L}$. The maximum of $\mathcal{L}$ defines the best fit parameter values, and contours offset by various $\Delta \mathcal{L}$ from $\mathcal{L}_{\text {max }}$ describe confidence limits for the parameters. Figure 2 shows the results of this 
analysis: while $v_{c}(-19,1)=85 \pm 8 \mathrm{~km} / \mathrm{s}$ is well constrained, the slopes $\eta$ and $\zeta$ are not. However, the trend that bluer galaxies have smaller linewidths (at fixed $b$-band luminosity), is significant at the $\geq 95 \%$ confidence limit. This analysis also reveals that all the scatter in Figure 1 is consistent with being due to the breadth of $p\left(\sigma / v_{c}\right)$ and due to the observational errors; the intrinsic scatter in the relation, $\Delta$, may be small.

\section{Results and Comparison with Local Samples}

As Figure 2 shows, these blue $\sim \mathrm{L}_{*} / 4$ galaxies at $z \sim 0.25$ have linewidths that correspond to circular velocities of $\sim 85 \mathrm{~km} / \mathrm{s}$. To test for evolution in the LWLC relation with cosmic epoch, we have to define the corresponding local relation. We have attempted to do so by using Tully-Fisher data for nearby galaxies of the same magnitude and color range (e.g. Bothun et al. 1985). After a $15 \%$ downward correction from $W_{20} /(2 \sin i)$ to the "optical" $v_{c}$, we find that the linewidths for the local galaxies are $15 \%$ larger than for the distant sample. For a relation $L_{b} \propto v_{c}^{3}$, this means that blue galaxies of the same linewidth were $\sim 50 \%$ more luminous at $z \sim 0.25$ than they are now.

We have presented a novel way to compare distant and local galaxy samples. However, at this point the analysis must be viewed as preliminary and the evolutionary inferences just reached should be taken with several grains of salt:

- Relating the observable $\sigma$ to $v_{c}$ requires a large and model dependent correction. This problem may be worst for a fibre measurement, where all spatial information is forsaken, but is likely to plague all ground-based observations with resolutions $\gtrsim 0.5^{\prime \prime}$. An important source of error could be eliminated by obtaining galaxy inclinations from HST imaging.

- Most local Tully-Fisher studies focus on galaxies with $L>\mathrm{L}_{*}$ and have morphological sample selection criteria which cannot be reproduced in the distant samples. Better samples for local comparison are needed.

- Detailed, but telescope time consuming, studies of the spatially resolved velocity fields in a few distant galaxies (ground-based or with STIS) will be extremely helpful in gauging the $p\left(\sigma / v_{c}\right)$ relation.

\section{References}

Bothun, G. et al., 1985, ApJS, 57, 423

Colless, M., Ellis, R., Broadhurst, T., Taylor, K., \& Peterson, B., 1993, MN, 261, 18.

Koo, D. and Kron, R., 1992, ARAA, 30, 613

Rix, H.-W., Guhathakurta, P., Colless, M. and Ing, K., 1996, MNRAS, to be submitted 